九州大学学術情報リポジトリ

Kyushu University Institutional Repository

\title{
SOME ALGEBRA FOR THE EXPONENTIAL-GAMMA PROCESS
}

Nadarajah, Saralees

School of Mathematics, University of Manchester

https://doi.org/10.5109/16774

出版情報: Bulletin of informatics and cybernetics. 39, pp.59-67, 2007-12. Research Association of Statistical Sciences

バージョン :

権利関係 : 
SOME ALGEBRA FOR THE EXPONENTIAL-GAMMA PROCESS

by

Saralees NADARAJAH

Reprinted from the Bulletin of Informatics and Cybernetics

Research Association of Statistical Sciences, Vol.39

FUKUOKA, JAPAN

2007 


\title{
SOME ALGEBRA FOR THE EXPONENTIAL-GAMMA PROCESS
}

\author{
By
}

Saralees NADARAJAH*

\begin{abstract}
The exponential-gamma (EG) process is a process made up of two random components $X$ and $Y$ : one exponentially distributed and the other gamma distributed. The EG process has attracted applications in almost every area of the sciences and engineering. In this note, we derive the exact distributions of $\alpha X+\beta Y, X Y$, and $X / Y$ and discuss how they are relevant to the applications of the EG process.
\end{abstract}

Key Words and Phrases: Exponential distribution, Gamma distribution, Linear combination of random variables, Product of random variables, Ratio of random variables.

\section{Introduction}

The exponential-gamma (EG) process has been a traditional model for many areas of the sciences and engineering. As the name implies, the process is composed of two random components: one exponentially distributed (say $X$ ) and the other gamma distributed (say $Y$ ). Usually, $X$ and $Y$ are assumed to be independent random variables. The composition of $X$ and $Y$ can take different forms depending on the nature of the application of the EG process. For example, in marketing sciences, the EG process is used to model repeat visiting behavior with individual's inter-visit times assumed to be exponentially distributed and governed by a rate that is gamma distributed across the population, see Morrison and Schmittlein (1988). This example clearly involves the product $X Y$. In production research, the EG process is used to describe the sequence of busy and idle periods. If one is interested in the inter arrival time (sometimes referred to as the cycle time) between idle periods or that between busy periods then that would entail the sum $X+Y$, see Nadarajah and Kotz (2006). For other related examples, see Park and Fader (2004) and Schroder (2004).

In this note, we derive the exact distributions of $\alpha X+\beta Y, X Y$, and $X / Y$ when $X$ and $Y$ are independent random variables with their probability density functions (pdfs) specified by

$$
f(x)=\lambda \exp (-\lambda x)
$$

and

$$
f(y)=\frac{\mu^{a} y^{a-1} \exp (-\mu y)}{\Gamma(a)},
$$

* School of Mathematics, University of Manchester, Manchester M60 1QD, UK. E-mail: saralees.nadarajah@manchester.ac.uk 
respectively, for $x>0, y>0, \lambda>0, \mu>0$ and $a>0$. We assume without loss of generality that $\alpha>0$. The calculations involve several special functions, including the modified Bessel function of the first kind defined by

$$
I_{\nu}(x)=\frac{x^{\nu}}{2^{\nu} \Gamma(\nu+1)} \sum_{k=0}^{\infty} \frac{1}{(\nu+1)_{k} k !}\left(\frac{x^{2}}{4}\right)^{k},
$$

the modified Bessel function of the third kind defined by

$$
K_{\nu}(x)=\frac{\pi\left\{I_{-\nu}(x)-I_{\nu}(x)\right\}}{2 \sin (\nu \pi)},
$$

the Airy function defined by

$$
A i(x)=\frac{\sqrt{x}}{\sqrt{3} \pi} K_{1 / 3}\left(\frac{2 x^{3 / 2}}{3}\right),
$$

the incomplete gamma function defined by

$$
\gamma(a, x)=\int_{0}^{x} t^{a-1} \exp (-t) d t,
$$

the complementary incomplete gamma function defined by

$$
\Gamma(a, x)=\int_{x}^{\infty} t^{a-1} \exp (-t) d t,
$$

and the error function defined by

$$
\operatorname{erfc}(x)=\frac{2}{\sqrt{\pi}} \int_{x}^{\infty} \exp \left(-t^{2}\right) d t,
$$

where $(e)_{k}=e(e+1) \cdots(e+k-1)=\Gamma(e+k) / \Gamma(e)$ denotes the ascending factorial with the convention $(e)_{0}=1$. The properties of the above special functions can be found in Prudnikov et al. (1986) and Gradshteyn and Ryzhik (2000).

\section{Exact Distribution of the Linear Combination}

Theorem 1 derives explicit expressions for the pdf and the cumulative distribution function (cdf) of $\alpha X+\beta Y$ in terms of the incomplete gamma functions.

Theorem 1 Suppose $X$ and $Y$ are independent random variables distributed according to (1) and (2), respectively. The cdf of $Z=\alpha X+\beta Y$ can be expressed as

$$
F_{Z}(z)=\frac{1}{\Gamma(a)} \gamma\left(a, \frac{\mu z}{\beta}\right)-\frac{(\mu \alpha)^{a}}{(\mu \alpha-\lambda \beta)^{a} \Gamma(a)} \exp \left(-\frac{\lambda z}{\alpha}\right) \gamma\left(a, \frac{z(\mu \alpha-\lambda \beta)}{\alpha \beta}\right)
$$

for $\beta>0$ and $z>0$, as

$$
F_{Z}(z)=\frac{1}{\Gamma(a)} \Gamma\left(a, \frac{\mu z}{\beta}\right)-\frac{(\mu \alpha)^{a}}{(\mu \alpha-\lambda \beta)^{a} \Gamma(a)} \exp \left(-\frac{\lambda z}{\alpha}\right) \Gamma\left(a, \frac{z(\mu \alpha-\lambda \beta)}{\alpha \beta}\right)
$$


for $\beta<0$ and $z<0$, and as

$$
F_{Z}(z)=1-\frac{(\mu \alpha)^{a}}{(\mu \alpha-\lambda \beta)^{a}} \exp \left(-\frac{\lambda z}{\alpha}\right)
$$

for $\beta<0$ and $z \geq 0$. The corresponding pdfs are:

$$
f_{Z}(z)=\frac{\lambda(\mu \alpha)^{a}}{\alpha(\mu \alpha-\lambda \beta)^{a} \Gamma(a)} \exp \left(-\frac{\lambda z}{\alpha}\right) \gamma\left(a, \frac{z(\mu \alpha-\lambda \beta)}{\alpha \beta}\right)
$$

for $\beta>0$ and $z>0$,

$$
f_{Z}(z)=\frac{\lambda(\mu \alpha)^{a}}{\alpha(\mu \alpha-\lambda \beta)^{a} \Gamma(a)} \exp \left(-\frac{\lambda z}{\alpha}\right) \Gamma\left(a, \frac{z(\mu \alpha-\lambda \beta)}{\alpha \beta}\right)
$$

for $\beta<0$ and $z<0$, and

$$
f_{Z}(z)=\frac{\lambda(\mu \alpha)^{a}}{\alpha(\mu \alpha-\lambda \beta)^{a}} \exp \left(-\frac{\lambda z}{\alpha}\right)
$$

for $\beta<0$ and $z \geq 0$.

PROOF. If $\beta>0$ then the result follows by writing

$$
\begin{aligned}
\operatorname{Pr}(\alpha X+\beta Y \leq z) & =\operatorname{Pr}\left(X \leq \frac{z-\beta Y}{\alpha}\right) \\
& =\int_{0}^{z / \beta} F_{X}\left(\frac{z-\beta y}{\alpha}\right) f_{Y}(y) d y \\
& =F_{Y}\left(\frac{z}{\beta}\right)-\frac{\mu^{a} \exp (-\lambda z / \alpha)}{\Gamma(a)} \int_{0}^{z / \beta} y^{a-1} \exp \left(-\frac{\mu \alpha-\lambda \beta}{\alpha} y\right) d y \\
& =\frac{1}{\Gamma(a)} \gamma\left(a, \frac{\mu z}{\beta}\right) \\
& \quad-\frac{\mu^{a} \exp (-\lambda z / \alpha)}{\Gamma(a)} \frac{\alpha^{a}}{(\mu \alpha-\lambda \beta)^{a}} \gamma\left(a, \frac{z(\mu \alpha-\lambda \beta)}{\alpha \beta}\right),
\end{aligned}
$$

where the last step follows from the definition of the incomplete gamma function. The result in (4) can be established similarly by using the definition of the complementary incomplete gamma function. The result in (5) follows by setting $z=0$ into to the two incomplete gamma function terms in (4).

The following corollaries provide the cdfs for the sum and the difference of the exponential and gamma random variables.

Corollary 1 Suppose $X$ and $Y$ are independent random variables distributed according to (1) and (2), respectively. Then, the cdf of $Z=X+Y$ can be expressed as

$$
F_{Z}(z)=\frac{1}{\Gamma(a)} \gamma(a, \mu z)-\frac{\mu^{a}}{(\mu-\lambda)^{a} \Gamma(a)} \exp (-\lambda z) \gamma(a, z(\mu-\lambda))
$$

for $z>0$. 
Corollary 2 Suppose $X$ and $Y$ are independent random variables distributed according to (1) and (2), respectively. Then, the cdf of $Z=X-Y$ can be expressed as

$$
F_{Z}(z)=\frac{1}{\Gamma(a)} \Gamma(a, \mu z)-\frac{\mu^{a}}{(\mu+\lambda)^{a} \Gamma(a)} \exp (-\lambda z) \Gamma(a, z(\mu+\lambda))
$$

for $z<0$ and as

$$
F_{Z}(z)=1-\frac{\mu^{a}}{(\mu+\lambda)^{a}} \exp (-\lambda z)
$$

for $z \geq 0$.

Using special properties of the incomplete gamma functions, one can obtain simpler expressions for (3)-(4) when $a$ takes integer or half integer values. This is illustrated in the corollaries below.

Corollary 3 If $a \geq 1$ is an integer then (3)-(4) can be reduced to the simpler forms

$$
\begin{gathered}
F_{Z}(z)=1-\exp (-y) \sum_{k=0}^{a-1} \frac{y^{k}}{k !}-\frac{(\mu \alpha)^{a}}{(\mu \alpha-\lambda \beta)^{a}} \exp \left(-\frac{\lambda z}{\alpha}\right) \\
+\frac{(\mu \alpha)^{a}}{(\mu \alpha-\lambda \beta)^{a}} \exp \left(-x-\frac{\lambda z}{\alpha}\right) \sum_{k=0}^{a-1} \frac{x^{k}}{k !}
\end{gathered}
$$

for $\beta>0$ and $z>0$, and

$$
F_{Z}(z)=\exp (-y) \sum_{k=0}^{a-1} \frac{y^{k}}{k !}-\frac{(\mu \alpha)^{a}}{(\mu \alpha-\lambda \beta)^{a}} \exp \left(-x-\frac{\lambda z}{\alpha}\right) \sum_{k=0}^{a-1} \frac{x^{k}}{k !}
$$

for $\beta<0$ and $z<0$, where $x=z(\mu \alpha-\lambda \beta) /(\alpha \beta)$ and $y=\mu z / \beta$.

Corollary 4 If $a-1 / 2 \geq 0$ is an integer then (3)-(4) can be reduced to the simpler forms

$$
\begin{aligned}
F_{Z}(z)=1- & \operatorname{erfc}(\sqrt{y})-\frac{(-1)^{a-3 / 2} \sqrt{y} \exp (-y)}{\Gamma(a)} \sum_{k=0}^{a-3 / 2}(1-a)_{a-k-3 / 2}(-y)^{k} \\
& -\frac{(\mu \alpha)^{a}}{(\mu \alpha-\lambda \beta)^{a}} \exp \left(-\frac{\lambda z}{\alpha}\right)+\frac{(\mu \alpha)^{a} \operatorname{erfc}(\sqrt{x})}{(\mu \alpha-\lambda \beta)^{a}} \exp \left(-\frac{\lambda z}{\alpha}\right) \\
& +\frac{(-1)^{a-3 / 2} \sqrt{x}(\mu \alpha)^{a} \exp (-x)}{(\mu \alpha-\lambda \beta)^{a} \Gamma(a)} \exp \left(-\frac{\lambda z}{\alpha}\right) \sum_{k=0}^{a-3 / 2}(1-a)_{a-k-3 / 2}(-x)^{k}
\end{aligned}
$$

for $\beta>0$ and $z>0$, and

$$
\begin{aligned}
F_{Z}(z)=\operatorname{erfc}( & \sqrt{y})+\frac{(-1)^{a-3 / 2} \sqrt{y} \exp (-y)}{\Gamma(a)} \sum_{k=0}^{a-3 / 2}(1-a)_{a-k-3 / 2}(-y)^{k} \\
& -\frac{(\mu \alpha)^{a} \operatorname{erfc}(\sqrt{x})}{(\mu \alpha-\lambda \beta)^{a}} \exp \left(-\frac{\lambda z}{\alpha}\right) \\
& -\frac{(-1)^{a-3 / 2} \sqrt{x}(\mu \alpha)^{a} \exp (-x)}{(\mu \alpha-\lambda \beta)^{a} \Gamma(a)} \exp \left(-\frac{\lambda z}{\alpha}\right) \sum_{k=0}^{a-3 / 2}(1-a)_{a-k-3 / 2}(-x)^{k}
\end{aligned}
$$


(a)

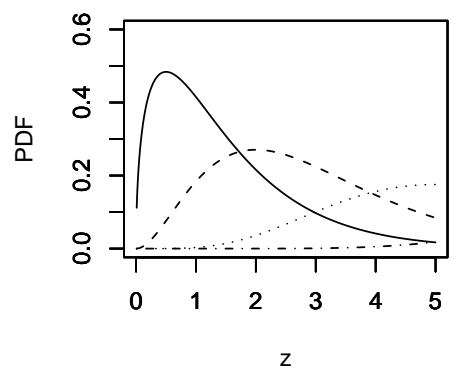

(c)

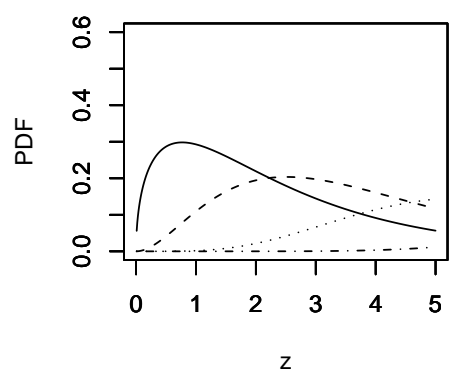

(b)

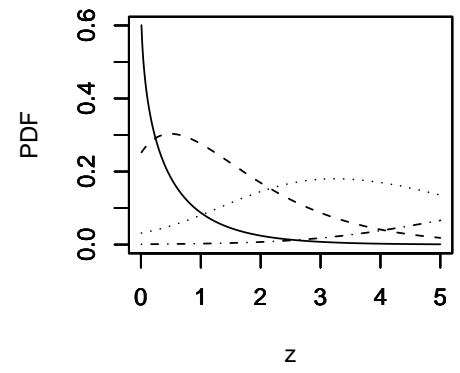

(d)

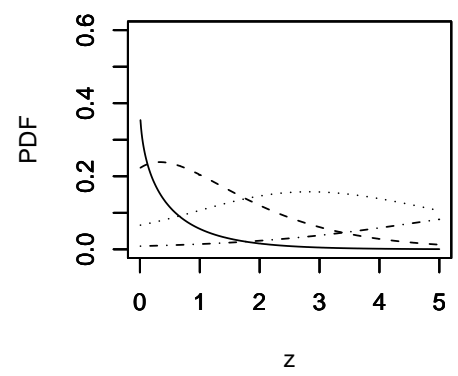

Figure 1: Plots of the pdf of (3)-(4) for $\lambda=1, \mu=1, a=0.5,2,5,10$, and (a): $\alpha=1$ and $\beta=1$; (b): $\alpha=1$ and $\beta=-1$; (c): $\alpha=1$ and $\beta=2$; and, (d): $\alpha=1$ and $\beta=-2$. The curves with the left to the right correspond to increasing values of $a$.

for $\beta<0$ and $z<0$, where $x=z(\mu \alpha-\lambda \beta) /(\alpha \beta)$ and $y=\mu z / \beta$. If $a=1 / 2$ then the terms containing the sum $\sum_{k=0}^{a-3 / 2}$ should be ignored.

Figure 1 illustrates possible shapes of the pdfs (6)-(8) for selected values of $\alpha, \beta$ and $a$. The four curves in each plot correspond to selected values of $a$. As expected, the densities are unimodal and the effect of the parameters is evident.

\section{Exact Distribution of the Product}

Theorem 2 derives an explicit expression for the cdf of $X Y$ in terms of the modified Bessel function of the third kind.

Theorem 2 Suppose $X$ and $Y$ are independent random variables distributed according to (1) and (2), respectively. The cdf of $Z=X Y$ can be expressed as

$$
F(z)=1-\frac{2(\lambda \mu z)^{a / 2}}{\Gamma(a)} K_{a}(2 \sqrt{\lambda \mu z})
$$


for $z>0$. The corresponding pdf is

$$
\begin{gathered}
f(z)=\frac{2(\lambda \mu)^{(a+1) / 2} z^{(a-1) / 2}}{\Gamma(a)}\left\{K_{a-1}(2 \sqrt{\lambda \mu z})+\frac{a}{2 \sqrt{\lambda \mu z}} K_{a}(2 \sqrt{\lambda \mu z})\right\} \\
-\frac{a(\lambda \mu)^{a / 2} z^{a / 2-1}}{\Gamma(a)} K_{a}(2 \sqrt{\lambda \mu z})
\end{gathered}
$$

for $z>0$.

Proof. The cdf corresponding to $(1)$ is $1-\exp (-\lambda x)$. Thus, one can write the cdf of $X Y$ as

$$
\begin{aligned}
\operatorname{Pr}(X Y \leq z) & =\int_{0}^{\infty} F_{X}(z / y) f_{Y}(y) d y \\
& =\int_{0}^{\infty}\left\{1-\exp \left(-\frac{\lambda z}{y}\right)\right\} \frac{\mu^{a} y^{a-1} \exp (-\mu y)}{\Gamma(a)} d y \\
& =1-\frac{\mu^{a}}{\Gamma(a)} \int_{0}^{\infty} y^{a-1} \exp \left(-\mu y-\frac{\lambda z}{y}\right) d y \\
& =1-\frac{\mu^{a}}{\Gamma(a)} I .
\end{aligned}
$$

Application of equation (3.471.9) in Gradshteyn and Ryzhik (2000) shows that the integral $I$ can be calculated as

$$
I=2\left(\frac{\lambda z}{\mu}\right)^{a / 2} K_{a}(2 \sqrt{\lambda \mu z}) .
$$

The result in (9) follows by substituting (12) into (11). The result of (10) follows by using the property $K_{\nu}^{\prime}(z)=-K_{\nu-1}(z)-(\nu / z) K_{\nu}(z)$.

Using special properties of the modified Bessel function of the third kind, one can obtain simpler expressions for the cdf of $X Y$ when $a-1 / 2, a-1 / 3$ or $a-2 / 3$ takes integer values. This is illustrated in the corollaries below.

Corollary 5 If $a-1 / 2 \geq 0$ is an integer then (9) can be reduced to the simpler form

$$
F(z)=1-\frac{\sqrt{\pi} x^{a} \exp (-x)}{2^{a-1 / 2} \Gamma(a) \sqrt{x}} \sum_{j=0}^{a-1 / 2} \frac{(j+a-1 / 2) !}{j !(-j+a-1 / 2) !(2 x)^{j}},
$$

where $x=2 \sqrt{\lambda \mu z}$.

Corollary 6 If $a-1 / 3 \geq 0$ is an integer then (9) can be reduced to the simpler form

$$
\begin{aligned}
F(z)=1 & -\frac{(-1)^{a-1 / 3} 2^{1 / 3} \pi \Gamma(-1 / 3)}{3^{5 / 6} \Gamma(a) \Gamma(1-a)} \\
& \times\left[3^{2 / 3} x^{2 / 3} \mathrm{Ai}^{\prime}\left((3 / 2)^{2 / 3} x^{2 / 3}\right)\right.
\end{aligned}
$$




$$
\begin{gathered}
\times \sum_{k=0}^{a-4 / 3} \frac{(a-k-4 / 3) !}{k !(a-2 k-4 / 3) !(4 / 3)_{k}(1-a)_{k}}\left(-\frac{x^{2}}{4}\right)^{k} \\
-2^{2 / 3} \operatorname{Ai}\left((3 / 2)^{2 / 3} x^{2 / 3}\right) \\
\left.\times \sum_{k=0}^{a-1 / 3} \frac{(a-k-1 / 3) !}{k !(a-2 k-1 / 3) !(1 / 3)_{k}(1-a)_{k}}\left(-\frac{x^{2}}{4}\right)^{k}\right],
\end{gathered}
$$

where $x=2 \sqrt{\lambda \mu z}$.

Corollary 7 If $a-2 / 3 \geq 0$ is an integer then (9) can be reduced to the simpler form

$$
\begin{aligned}
F(z)=1- & \frac{(-1)^{a+1 / 3} \pi \Gamma(-2 / 3)}{2^{1 / 3} 33^{5 / 6} \Gamma(1-a) \Gamma(a)} \\
& \times\left[9 x^{4 / 3} \mathrm{Ai}\left((3 / 2)^{2 / 3} x^{2 / 3}\right)\right. \\
& \quad \times \sum_{k=0}^{a-5 / 3} \frac{(a-k-5 / 3) !}{k !(a-2 k-5 / 3) !(5 / 3)_{k}(1-a)_{k}}\left(-\frac{x^{2}}{4}\right)^{k} \\
& -42^{1 / 3} 3^{2 / 3} \mathrm{Ai}^{\prime}\left((3 / 2)^{2 / 3} x^{2 / 3}\right) \\
& \left.\quad \times \sum_{k=0}^{a-2 / 3} \frac{(a-k-2 / 3) !}{k !(a-2 k-2 / 3) !(2 / 3)_{k}(1-a)_{k}}\left(-\frac{x^{2}}{4}\right)^{k}\right],
\end{aligned}
$$

where $x=2 \sqrt{\lambda \mu z}$.

Figure 2 illustrates possible shapes of the pdf of (9) for selected values of $a$. As expected, the densities are unimodal and the effect of the parameter is evident.

\section{Exact Distribution of the Ratio}

Theorem 3 derives an elementary expression for the cdf of $X / Y$.

Theorem 3 Suppose $X$ and $Y$ are independent random variables distributed according to (1) and (2), respectively. The cdf of $Z=X / Y$ can be expressed as

$$
F(z)=1-\frac{\mu^{a}}{(\mu+\lambda z)^{a}}
$$

for $z>0$. The corresponding pdf is

$$
f(z)=\frac{a \lambda \mu^{a}}{(\mu+\lambda z)^{a+1}}
$$

for $z>0$.

Proof. The result follows by writing

$$
\operatorname{Pr}(X / Y \leq z)=\int_{0}^{\infty} F_{X}(z y) f_{Y}(y) d y
$$




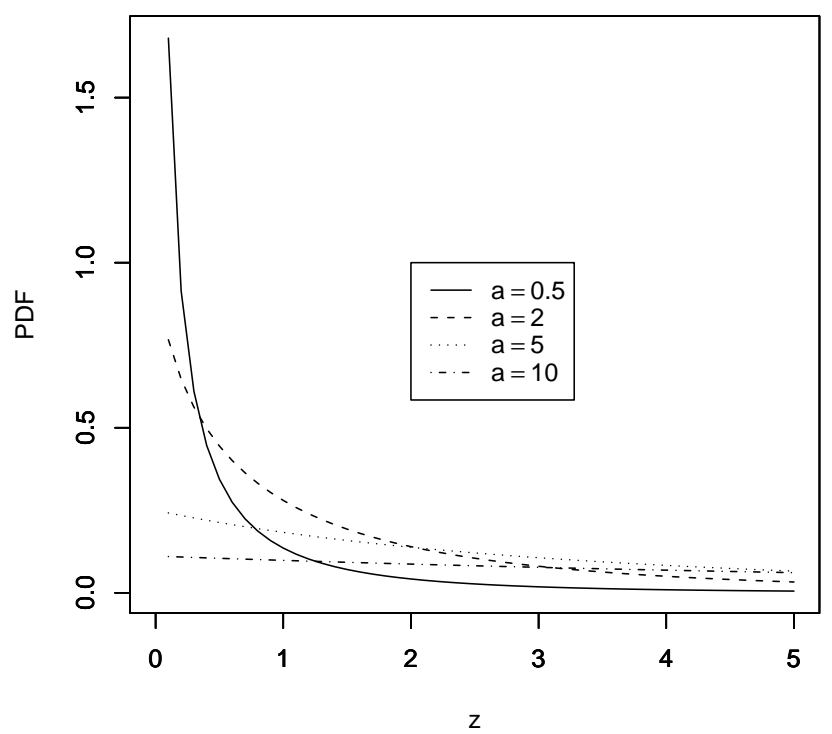

Figure 2: Plots of the pdf of (9) for $\lambda=1, \mu=1$ and $a=0.5,2,5,10$.

$$
\begin{aligned}
& =\int_{0}^{\infty}\{1-\exp (-\lambda y z)\} \frac{\mu^{a} y^{a-1} \exp (-\mu y)}{\Gamma(a)} d y \\
& =1-\frac{\mu^{a}}{\Gamma(a)} \int_{0}^{\infty} y^{a-1} \exp \{-(\mu+\lambda z) y\} d y \\
& =1-\frac{\mu^{a}}{(\mu+\lambda z)^{a}} .
\end{aligned}
$$

The proof is complete.

Note that the distribution given by (13) is the well-known $F$ distribution.

\section{References}

Gradshteyn, I. S. and Ryzhik, I. M. (2000). Table of Integrals, Series, and Products, sixth edition, Academic Press, San Diego.

Morrison, D. G and Schmittlein, D. C. (1988). Generalizing the NBD model for customer purchases: what are the implications and is it worth the effort? Journal of Business and Economic Statistics 6, 145-159.

Nadarajah, S. and Kotz, S. (2007). The cycle time distribution, International Journal of Production Research, in press.

Park, Y. H. and Fader, P. S. (2004). Modeling browsing behavior at multiple websites, Marketing Science 23, 280-303. 
Prudnikov, A. P., Brychkov, Y. A. and Marichev, O. I. (1986). Integrals and Series, volume 1, Gordon and Breach Science Publishers, Amsterdam.

M. Schroder (2004). Risk-neutral parameter shifts and derivatives pricing in discrete time, The Journal of Finance 59, 2375-2402.

Received April 19, 2007

Revised August 24, 2007 